\title{
DERECHO Y POLÍTICAS AMBIENTALES EN CASTILLA-LA MANCHA (SEGUNDO SEMESTRE 2016)
}

\author{
Nuria María GaRrido Cuenca \\ Profesora Titular Derecho Administrativo \\ Universidad de Castilla-La Mancha
}

\begin{abstract}
Sumario: 1. Introducción. De nuevo las polémicas en torno al ATC de Villar de Cañas y la política hidráulica de trasvases. Las dudas sobre la gestión ambiental de los residuos a raíz de los incendios de Seseña y Chiloeches. 2. Medidas relativas a la conservación de la naturaleza. En particular, la ampliación de la ZEPA de la Laguna del Hito y de nuevo las controversias en torno al ATC de Villar de Cañas. 3. Medidas en el ámbito tributario: la revisión de las principales tasas ambientales. 4. Caza y pesca. 5. Medidas de fomento. 6. Consecuencias medioambientales de un periodo marcado por los trágicos incendios de Seseña y Chiloeches. 7. La continuidad de la política de trasvases y la oposición del Gobierno regional. 8. Actuaciones pendientes: reconversión de las minas de mercurio de Almadén y el conflicto en torno a la minería de tierras raras.
\end{abstract}

\section{Introducción. De nuevo las polémicas en torno al ATC de Villar de Cañas y la} política hidráulica de trasvases. Las dudas sobre la gestión ambiental de los residuos a raíz de los incendios de Seseña y Chiloeches

En el periodo analizado han sido escasas las normas de contenido ambiental sustantivo aprobadas, salvo las modificaciones en materia tributaria de la Ley autonómica de acompañamiento a los Presupuestos de 2016 que han modificado la práctica totalidad de las tasas ambientales vigentes, la publicación de las Ordenes de veda de caza y pesca y algunas ayudas como medidas de fomento. No obstante, sí debemos señalar la trascendencia que puede tener la decisión del Gobierno regional de ampliar la ZEPA de la Laguna del Hito, en la provincia de Cuenca, de modo que ha quedado especialmente protegida con este instrumento de política ambiental justo una de las zonas donde se está dilucidando la ubicación del Almacenamiento Temporal de Residuos Radioactivos, en Villar de Cañas, uno de los términos municipales afectados por la ampliación. La polémica no se ha hecho esperar, y tras el impasse electoral habrá que ver si el Gobierno Nacional continúa insistiendo en el interés general de este ATC y si recurre el Decreto autonómico sobre la ZEPA. Mientras tanto la sociedad se encuentra bastante dividida, con plataformas ciudadanas a favor y en contra de esta medida, resultando interesante la necesaria ponderación de intereses en conflicto, económicos, ambientales y de seguridad 
que seguramente terminarán ventilándose ante los Tribunales.

Al margen de las medidas de orden normativo, este periodo ha estado marcado por dos incendios en Toledo y Guadalajara, en sendos almacenamientos de neumáticos y residuos peligrosos, que crearon enorme alarma social y que han tenido nefastas consecuencias medioambientales. La apertura de sendos procedimientos judiciales para la averiguación de los hechos y la depuración de responsabilidades está siendo especialmente intensa, dejando al descubierto importantes déficits en el modelo de gestión de los residuos en nuestra Comunidad Autónoma.

En fin, como en la Crónica anterior ya exponíamos, no podemos dejar de referirnos nuevamente a la política hidráulica del Gobierno estatal, que nuevamente ha aprobado dos triples trasvases de agua al Levante y además ha ampliado el Decreto de sequía cuya vigencia terminaba en el mes de octubre por un año más, esquilmando más si cabe las perspectivas de nuestros agricultores y nuestros cultivos.

\section{Medidas relativas a la conservación de la naturaleza. En particular, la ampliación} de la ZEPA de la Laguna del Hito y de nuevo las controversias en torno al ATC de Villar de Cañas

En esta materia se ha producido, en primer lugar, una mínima modificación del Catálogo Regional de Especies Amenazas (contenido en el Decreto 33/1998, de 5 de mayo), que lleva a cabo el Decreto 22/2016 de 10 de mayo (DOCM 13 de mayo) para excluir la especie Cormorán grande (Phalacrocorax carbo sinensis), de acuerdo a los datos favorables de su incremento poblacional y su situación de conservación favorable. Con esta medida se adecúa nuestro registro al Catálogo Nacional que ya la excluyó en el Real Decreto 139/2011, de 4 de febrero.

Pero sin duda, la norma más relevante del periodo que analizamos es el Decreto 57/2016, de 4 de octubre por el que se amplía la Zona de Especial Protección para las Aves (ZEPA) Laguna de El Hito y se realiza la propuesta a la Comisión Europea para su declaración como Lugar de Importancia Comunitaria (LIC) (DOCM de 11 de octubre). Con esta norma se amplía la ZEPA en los términos municipales de la provincia de Cuenca de Almonacid del Marquesado, El Hito, Huete, Montalbo, Saelices, Villar de Cañas, Villarejo de Fuentes, Villares del Saz, y Zafra de Záncara, todos ellos de la provincia de 
Cuenca, como se recoge en el Anexo IV del Decreto. De este modo, esta zona de especial protección tendrá la consideración de espacio protegido a nivel estatal (art.41 de la Ley 42/2007, de 13 de diciembre, del Patrimonio Natural y de la Biodiversidad) y pasará a formar parte de la Red Regional de Áreas Protegidas como “zona sensible”, de acuerdo al art.54 de la Ley autonómica 9/1999, de 26 de mayo, de Conservación de la Naturaleza. Los cuatro objetivos de esta ampliación son: la mejor de la conservación del hábitat y la reducción de las presiones y amenazas sobre las especies de aves presentes y de las especies de aves migratorias que utilizan el espacio en la época de reproducción, invernada o durante los pasos migratorios; mantener y promover los aprovechamientos tradicionales agrarios; y mejorar la protección de los tipos de hábitat y las especies de flora y fauna de interés comunitario, especialmente aquellos calificados como prioritarios en la Directiva 92/43/CEE, relativa a la conservación de los hábitats naturales y de la fauna y flora silvestres. Justamente este último objetivo conduce a la proposición a la Comisión Europea para que se declare la ampliación de la Zona como lugar de importancia comunitaria (LIC).

Esta norma ha generado bastante polémica, pues la ampliación de la ZEPA conlleva la imposibilidad de seguir adelante con el proyecto estatal de ubicación del ATC (almacén temporal centralizado de combustible nuclear y residuos radioactivos) en Villar de Cañas, uno de los términos municipales afectados por la norma. Como ya dimos cuenta en las Crónicas pasadas (enero-junio de 2016), el Gobierno central impugnó el Acuerdo del Consejo de Gobierno autonómico de 28 de julio de 2015 por el que se iniciaba el procedimiento de ampliación del espacio protegido que ahora culmina la norma que analizamos, solicitando la medida cautelar de suspensión. El Tribunal Superior de Justicia de Castilla La Mancha desestimó esta petición en su Auto de 22 de diciembre de 2015, quedando todavía pendiente la resolución del fondo del asunto. Recordemos que, en síntesis, el Tribunal entenderá prevalentes los intereses ambientales que podrían verse afectados de forma irreparable frente a los puramente económicos que había esgrimido la Administración estatal. Además, los informes del Consejo de Seguridad Nuclear (CSN), en principio secretos y confidenciales, que fueron publicados a raíz de la decisión del Consejo de Transparencia de 27 de febrero de 2016, demostraron que los terrenos no eran los más adecuados ni idóneos como ubicación del cementerio nuclear en lid. Estudios posteriores insisten en la posible afección ambiental de los suelos salinos y yesosos propios de la zona, que han permitido la existencia de especies únicas y su valor 
excepcional como hábitat natural para las aves.

Sin embargo, la polémica se ha agudizado en el entorno de la zona, con intereses contradictorios entre quienes quieren hacer prevalecer el interés ambiental de la zona, quienes ven las posibilidades de crecimiento económico, los agricultores que temen una restricción en su actividad agraria y la propia Administración estatal que ha dejado en suspenso la ubicación del ATC. La Plataforma de afectados por la ampliación de la ZEPA sigue manteniendo la nulidad de la norma autonómica, insistiendo en la existencia de un fraude de ley, debido a la utilización de la normativa medioambiental, "para la obtención de un fin distinto del de la protección o conservación de la naturaleza y las especies, esto es, impedir la construcción del ATC". Habrá que esperar, en consecuencia, a que se diriman judicialmente las controversias, sobre todo desde el punto de vista competencial, entre los intereses generales económicos y los intereses ambientales puestos en valor por nuestras Administraciones.

\section{Medidas en el ámbito tributario: la revisión de las principales tasas ambientales}

La Ley 3/2016, de 5 de mayo de medidas tributarias y administrativas de Castilla La Mancha (DOCM de 11 de mayo), de acompañamiento a la Ley de Presupuestos, modifica en su mayor parte las tasas de la Consejería de Agricultura, Medio Ambiente y Desarrollo Rural reguladas en el capítulo IV del título IV de la Ley 9/2012, de 29 de noviembre, afectando a veinticuatro de las treinta y siete secciones en las que se recogen. El artículo 12 de la norma incorpora estas novedades respecto a la regulación anterior:

- Respecto a las tasas por autorización ambiental integrada, tramitación de comunicación de su modificación no sustancial y tramitación de la modificación sustancial se incorpora una exención en el pago de las mismas en favor de las entidades y órganos pertenecientes a la Administración de la Junta de Comunidades de Castilla-La Mancha y de otras administraciones públicas;

- Se suprimen las tasas por renovación, actualización o revisión de autorización ambiental integrada; por inscripción registral de organismo de control autorizado en el área de atmósfera; por inscripción registral en un sistema de gestión y auditoría medioambientales (EMAS); por renovación de la inscripción registral EMAS; por inscripción registral de equipamientos de educación ambiental; por suministro de 
información ambiental y por emisión de informes de evaluación de impacto ambiental, que quedan sin contenido;

- Se modifica el hecho imponible de la tasa por modificación del plan de seguimiento de gases de efecto invernadero, si lo es por cambios de actividad o en el método de medición;

- Respecto a los tributos relacionados con la gestión de residuos, se modifican la denominación y algunos elementos y se incorporan en una tasa única la autorización de instalación de gestión de residuos, autorización de gestor de residuos o autorizaciones conjuntas de instalación y gestor. Con esta modificación se da cumplimiento al art. 27.3 de la Ley 22/2011, de 28 de julio, de residuos y suelos contaminados, en los casos en los que la persona física o jurídica que solicite la autorización para realizar una o varias operaciones de tratamiento de residuos sea, a su vez, titular de la instalación de tratamiento donde van a desarrollarse dichas operaciones, en cuyo caso, el órgano ambiental competente concederá una sola autorización que comprenderá la de la instalación y la de las operaciones de tratamiento. Lo que conlleva también la modificación de algunos elementos de la que ahora pasan a denominarse tasa por modificaciones o ampliaciones de la autorización de instalación de gestión de residuos, tasa por autorización de sistemas de responsabilidad ampliada del productor de residuos, o tasa por prórroga o modificación de la autorización de sistemas de responsabilidad ampliada del productor de residuos. Asimismo se ha modificado la cuota tributaria de la tasa por autorización de la modificación de la duración de almacenamiento de residuos peligrosos, incorporando determinadas exenciones para las autoridades administrativas, para adaptarla a las disposiciones de naturaleza básica de la Disposición Adicional octava de la Ley 21/2013 de 9 de diciembre, de evaluación ambiental;

- La autorización de instalación potencialmente contaminadora de la atmósfera se ha modificado sustancialmente, cambiando el hecho imponible, los sujetos pasivos e incorporándose una exención en favor de las entidades y órganos administrativos;

- En fin, se modifica la cuota tributaria de la tasa por autorización de entidades y profesionales para el seguimiento de puntos críticos de la declaración de impacto ambiental. 


\section{Caza y pesca}

La Orden de 7 de junio de 2016 (DOCM 10 de junio) fija los periodos hábiles de caza y las vedas aplicables con carácter general en todo el territorio de Castilla La Mancha, en aplicación de Ley 3/2015, de 5 de marzo, de Caza de Castilla-La Mancha (art.59.1). En tanto todavía no se ha dictado el reglamento ejecutivo de esta Ley, siguen siendo de aplicación las disposiciones del Reglamento general de aplicación de la anterior Ley 2/1993, de Caza. Por ello, la nueva Orden de Vedas contempla los aspectos básicos relativos a: relación de especies susceptibles de caza, fijación de periodos y días de caza, establecimiento de posibles medidas circunstanciales para protección o control de las poblaciones cinegéticas y limitaciones o excepciones.

Las principales novedades de esta norma para la temporada cinegética 2016/2017, es la exclusión de determinadas especies cinegéticas, por no tener en nuestra Región poblaciones que permitan su aprovechamiento cinegético sostenible: Cerceta carretona, Ánade silbón, Porrón común, Porrón moñudo, Agachadiza común, Agachadiza chica, Gaviota patiamarilla, Gaviota reidora (Larus ridibundus) y Grajilla. Por otro lado, y con el fin de proteger las poblaciones de tórtola común, al igual que en el año anterior, se establece un cupo máximo de ejemplares a abatir por cazador y día.

También se incluyen limitaciones a la caza de Becada, con el fin de garantizar el aprovechamiento sostenible de la especie y se autoriza la caza de perdiz roja con reclamo, al amparo del nuevo art.27.n) de la Ley 3/2015 de Caza. En fin, aunque la caza de los machos de corzo en el año 2017 comienza el 1 de abril, también se prevé su regulación en la norma, así como el periodo para la caza de las hembras de esta especie en aquellos cotos que lo tengan autorizado.

Por su parte, la Orden de 23 de junio de 2016, de Vedas de pesca (DOCM de 5 de julio) modifica la Orden de 19 de enero, de la Consejería de Agricultura, Medio Ambiente y Desarrollo Rural de Vedas de Pesca de 2016, de la que se dio cuenta en la Crónica anterior (DOCM de 28 de enero). Esta revisión obedece al necesario cumplimiento de la Sentencia del Tribunal Supremo 637/2016, de 16 de marzo, que estimó parcialmente el recurso contencioso-administrativo contra el Real Decreto 630/2013, de 2 de agosto, por el que se regula el Catálogo español de especies exóticas invasoras. En esta decisión judicial se declaró la nulidad de la exclusión del Catálogo de las especies carpa común (Cyprinus carpio) y la trucha arcoíris (Oncorhynchus mykiss), la exclusión del ámbito de aplicación de la norma de la comercialización del cangrejo rojo destinado a la industria 
alimentaria, y en fin de la disposición transitoria segunda de la norma estatal, que calificaba expresamente a las actividades cinegéticas y piscícolas como herramientas necesarias para la gestión y control de las especies exóticas invasoras incluidas en el Catálogo de especies invasoras.

La Orden de 23 de junio modifica sustancialmente la norma anterior en varios sentidos:

- En primer lugar, respecto a la comercialización de peces y cangrejos se establece que únicamente podrán comercializarse la anguila y la trucha arco iris, esta última solo con destino a la industria agroalimentaria;

- Respecto a los medios de pesca, al objeto de prevenir la introducción involuntaria de especies alóctonas acuáticas (mejillón cebra, almeja asiática, moco de roca...), se recomienda limpiar, secar $\mathrm{y}$, cuando sea posible, desinfectar cualquier tipo de embarcación, "patos", y cualquier otro medio utilizado para la pesca, en particular los reteles, rejones, así como los vadeadores y botas, antes de utilizarlos en aguas diferentes;

- Con el objeto de evitar la introducción de especies exóticas en las aguas de la Región, se establece en el Anexo I de la norma los ejemplares que, en caso de captura, no pueden devolverse a las aguas, debiendo dárseles muerte inmediata, excepto cuando se capturen por razones de investigación, salud o seguridad de las personas, previamente autorizada por la Consejería. Además sólo podrán pescarse en las áreas de distribución establecidas en el anexo III de la Orden, en el marco de estrategias para su gestión, control y posible erradicación;

- Con el fin de no perturbar la nidificación de las aves acuáticas, para la realización del control de poblaciones del cangrejo rojo se fija un período de veda comprendido entre el 1 de febrero y el 31 de mayo;

- Finalmente, se establece expresamente que la inclusión de una especie en el Catálogo español de especies exóticas invasoras conlleva la prohibición de su introducción en el medio natural. 


\section{Medidas de fomento}

La Orden de 20 de julio de 2016, de la Consejería de Agricultura, Medio Ambiente y Desarrollo Rural que establece las bases reguladoras de las ayudas para la ejecución de tratamientos silvícolas en el marco del Programa de Desarrollo Rural de Castilla-La Mancha 2014-2020 (DOCM no 146 de 27 de julio de 2016), de acuerdo a la normativa europea del Fondo Europeo Agrícola de Desarrollo Rural (FEADER). La convocatoria de estas ayudas se publica en la Resolución de 19 de septiembre de 2016, de la Dirección General de Política Forestal y Espacios Naturales (BOCM nº189, 27 de septiembre). Su objetivo es satisfacer las demandas de la sociedad en lo relativo a bienes y servicios ambientales potenciando al mismo tiempo la cubierta forestal arbórea, como medio para garantizar su funcionalidad social, protectora, productora y ecológica y que puedan permitir a sus propietarios alguna renta económica compatible con los principios de sostenibilidad de las masas forestales. Estas inversiones están destinadas a fomentar la multifuncionalidad de los montes, principio básico de la selvicultura por el cual se reconocen todas las utilidades posibles que ofrecen los terrenos forestales al conjunto de la sociedad, tanto aquellas sin rendimiento económico directo, también denominadas externalidades, como otras de carácter productivo. Así, como explicita la norma de convocatoria, el objeto principal de estas ayudas será el de aumentar la capacidad de adaptación y el valor medioambiental de los ecosistemas forestales al mejorar su estructura forestal mediante actuaciones encaminadas a conservar y mejorar la biodiversidad existente, evitar la degradación del suelo forestal y mantener la regulación hídrica. Además, con estas acciones se favorece la captura de carbono y se mejora el paisaje, a la vez que se facilita la creación y el mantenimiento de pequeñas empresas en el medio rural.

Por otro lado, la Orden de 18 de mayo de 2016 establece las bases reguladoras de las ayudas, en régimen de mínimos, para prevenir los posibles ataques de lobo ibérico al ganado doméstico en territorios de Castilla-La Mancha (DOCM de 23 de mayo). Esta norma, como otras de las que venimos dando noticia en Crónicas anteriores (desde la Orden de 23 de diciembre de 2003), tiene por fin la conciliación de la protección de esta especie actualmente catalogada en peligro de extinción con la reacción social frente a los daños causados en las explotaciones ganaderas que, en muchos casos, no disponen de medidas de defensa adecuadas, o donde las características de las especies ganaderas o los sistemas de manejo del ganado no son los adecuados para evitar los daños. 
Esa norma presenta como novedad que, a diferencia de las anteriores ayudas financiadas íntegramente por la Comunidad Autónoma, cuenta con la cofinanciación del Fondo de Patrimonio Natural previsto en la Ley estatal 42/2007, del Patrimonio Natural y la Biodiversidad, para el apoyo a medidas destinadas a prevenir o mitigar los daños provocados por determinadas especies protegidas, entre las que se cuenta el lobo ibérico. Sin embargo, se mantiene y mejora el régimen de ayudas previsto para la prevención de los ataques, subvencionando el apoyo a la instalación de vallados o arreglo de corrales y apriscos para mejorar las condiciones de seguridad del ganado frente a los ataques del lobo y la adquisición de mastines de guarda del ganado, previsto en la Orden de 26 de diciembre de 2014, que fue modificada por Orden de 12 de abril de 2016 (vid comentario en Crónica anterior).

\section{Consecuencias medioambientales de un periodo marcado por los trágicos incendios de Seseña y Chiloeches}

El periodo que comentamos ha sido especialmente trágico en incendios, no solo forestales, que han causado importantes daños medioambientales, trascendiendo la alarma social fuera del territorio de Castilla La Mancha. Los dos más importantes han tenido lugar en Seseña (Toledo) en un vertedero ilegal de neumáticos y en Chiloeches (Guadalajara) en una nave de disolventes. La magnitud de los costes de limpieza y restauración ambiental alcanza los 3 millones de euros en el primer caso y los 6 millones en el segundo, que serán repercutidos a los propietarios de las respectivas parcelas. Además, dado que una de las partes que se ha restaurado ambientalmente, en principio por la Junta de Comunidades, es dominio público hidráulico, dependiente de la Confederación Hidrográfica del Tajo, parte de ese coste será aportado por el Ministerio de Agricultura.

Sobre ambos incendios se han abierto los consecuentes procesos judiciales, en el de Seseña por el Juzgado madrileño de Valdemoro y el de Chiloeches por el Juzgado de Guadalajara. El desarrollo de los acontecimientos en este segundo caso ha puesto al descubierto la clandestinidad de este vertedero, que venía siendo advertida e investigada desde el Seprona por supuestos delitos medioambientales desde el año 2010. Primero por las irregularidades en el tratamiento de los residuos y segundo por la actividad de gestión de las millones de toneladas de residuos sin autorización ambiental en vigor (competencia 
de la Junta de Comunidades) ni licencia municipal. De hecho, el Ayuntamiento de Chiloeches decidió el pasado 17 de junio clausurar la planta de reciclaje de productos químicos por "actividad clandestina" y por "no disponer de licencia de apertura o autorización de puesta en funcionamiento para el ejercicio de su actividad", así como por "incumplimiento de normativas medioambientales y de seguridad", como recoge el BOE de 14 de julio de 2016. El actual Gobierno autonómico decretó en julio la suspensión temporal de la autorización para gestionar residuos de la empresa, aunque previamente ya se había suspendido esta autorización, que incluso fue respaldada por el Tribunal Superior de Justicia de Castilla La Mancha, en diciembre de 2014, sin que la empresa propietaria de las instalaciones KUK Medio Ambiente S.A. suspendiera realmente la actividad. En la causa penal abierta, también se ha personado la Guardia Civil, que acusa al exalcalde de la localidad -de los años 2011 a 2015- de "prevaricación ambiental" (art.329 Código Penal) por haber dejado continuar conscientemente la actividad de gestión de residuos.

Lo que resulta evidente es que, al margen de la solución judicial de estos casos, las irregularidades cometidas y la inactividad de las Administraciones competentes hasta solo dos meses antes de este incendio han demostrado que los instrumentos administrativos de gestión y control en materia de residuos no han funcionado correctamente. Así como el Plan Territorial de Emergencia de Castilla La Mancha (Platecam), respecto a la gestión del incendio.

\section{La continuidad de la política de trasvases y la oposición del Gobierno regional}

Nuevamente en este periodo debemos referirnos a varias normas estatales referidas a la política hidráulica que inciden directamente en nuestra Comunidad Autónoma. Si en la Crónica anterior ya esbozábamos las consecuencias nefastas de la revisión de la planificación hidrológica llevada a cabo por el Real Decreto 1/2016, de 8 de enero, así como el recurso aprobado por nuestro Gobierno regional contra el "triple trasvase" del Tajo al Segura de 60 hectómetros cúbicos para los meses de marzo a mayo y contra el trasvase de 10 hectómetros cúbicos de febrero, en este periodo se han vuelto a aprobar por el Ejecutivo estatal otros dos trasvases de igual envergadura y además se ha prorrogado, sorpresivamente, el Real Decreto de sequía en las demarcaciones hidrográficas del Júcar y del Segura hasta septiembre de 2017. 
Comenzando por la primera de las normas estatales, el Ministerio de Agricultura, Alimentación y Medio Ambiente ha autorizado sin solución de continuidad dos trasvases de 60 hectómetros cúbicos de agua cada uno para los trimestres de julio a septiembre y de octubre a diciembre, a través del acueducto Tajo-Segura. El primero de estos se realiza de acuerdo a la Orden AAA/1099/2016, de 6 de julio, por la que se autoriza un trasvase desde los embalses de Entrepeñas-Buendía, a través del acueducto Tajo-Segura, para los meses de julio, agosto y septiembre de 2016, a razón de 20 hm³/mes (BOE de 8 de julio), constatada la situación hidrológica excepcional, nivel 3, en el mes de julio de 2016, y la previsión de su mantenimiento durante los próximos meses. El segundo, por Orden AAA/1589/2016, de 3 de octubre, por la que se autoriza un trasvase de $60 \mathrm{hm}^{3}$ desde los embalses de Entrepeñas-Buendía, a través del acueducto Tajo-Segura, para el trimestre de octubre, noviembre y diciembre de 2016 (BOE 5 de octubre). El objetivo de este último, declarado en su Preámbulo, es poder atender las necesidades hídricas de las cuencas receptoras, garantizar todos los suministros de agua de la cuenca cedente, así como el abastecimiento de 2,5 millones de personas de las provincias de Murcia, Alicante y Almería. De acuerdo con el régimen transitorio previsto en la Ley de Evaluación Ambiental, en 2016 los trasvases son legalmente posibles en tanto las reservas de la cabecera del Tajo (Entrepeñas y Buendía) no estén por debajo del umbral de 336 Hm3. El pasado 1 de octubre, fecha de comienzo del nuevo año hidrológico, estos embalses almacenaban un total de 439,18 hm3 de agua.

Esta nueva derivación ha supuesto la oposición frontal del Gobierno castellanomanchego, que ha anunciado un nuevo recurso ante la Audiencia Nacional frente al trasvase. Los once trasvases mensuales aprobados, dos de ellos midiendo los datos aventuradamente con dos meses de antelación y basados en cuantías de máximos supone una actuación "ilegal” y “temeraria”. Como se ha señalado por el Ejecutivo autonómico, según las propias mediciones estatales que autorizan el trasvase, no se ha tenido en cuenta el descuento de 60 Hectómetros cúbicos que se derivarán al Segura, y que harían descender por debajo de los 400 hectómetros, línea roja que separa el nivel 3 de situación excepcional del nivel 4, que impediría la autorización de cualquier trasvase. La consejera ha recordado que la entrada en vigor de este umbral para no poder trasvasar de 400 hectómetros cúbicos lo reconoció el propio Ministerio de Agricultura en un comunicado público emitido el 8 de marzo de 2014 y en un certificado remitido a la Agencia Regional del Agua de Castilla-La Mancha desde la Confederación Hidrográfica del Tajo. Pese a 
ello, el Ministerio persiste desde el verano pasado en actuar contra sus propios actos jurídicos y sitúa la línea mínima entre ambos niveles en 336 hectómetros cúbicos para este año 2016.

El ritmo de pérdida de agua de los embalses de cabecera es otra de las razones que inducen a pensar que el nivel de los pantanos llegará a ser inferior a los 400 hectómetros. Sólo cinco días después de la medición de 439 hectómetros tomada como referencia para autorizar el nuevo trasvase triple, la suma de Entrepeñas y Buendía es de 436 hectómetros. Esa pérdida de agua, a razón de prácticamente un hectómetro cúbico diario, refleja un alto índice de evaporación a consecuencia de un clima inusual para el otoño, lo que hace prever una acusada curva de descenso del agua almacenada en la cabecera. Además de las previsiones climatológicas a más largo plazo de un otoño excepcionalmente seco y las temperaturas mucho más elevadas de lo normal.

Además de estas medidas, el Ministerio ha dictado el Real Decreto 335/2016, de 23 de septiembre, por el que se prorroga la situación de sequía declarada para el ámbito territorial de la Confederación Hidrográfica del Júcar por el Real Decreto 355/2015, de 8 de mayo, y para el ámbito territorial de la Confederación Hidrográfica del Segura por el Real Decreto 356/2015, de 8 de mayo (BOE de 24 de septiembre) hasta septiembre de 2017. Dicha prorroga permitirá seguir aplicando durante la campaña de riego medidas excepcionales para paliar los efectos de escasez de precipitaciones en ambas cuencas hidrográficas, siempre a favor del Levante español, a pesar de que el $25 \%$ de la cuenca del Segura pertenece a la provincia de Albacete, quien no podrá recurrir a las aguas subterráneas durante un año más y sin tener en cuenta el sudeste de Castilla-La Mancha muy afectado por la escasez de lluvias.

Como ya señalábamos en la Crónica del anterior periodo, la política hidráulica nacional sigue aumentando las limitaciones sobre el uso y disfrute de los recursos hídricos de Castilla La Mancha, favoreciendo a otras regiones. De modo que nuevamente habrá que esperar a un pronunciamiento judicial, que seguramente llegará tarde, para verificar la adecuación a la constitucionalidad y la legalidad de estas medidas que tanto afectan a nuestra región.

Mientras tanto, y ya cerrando esta crónica, se han desatado todas las alarmas sobre el estado de conservación de las aguas del río Tajo a raíz de la presencia de vertidos a su paso por la ciudad de Toledo. Nuevamente la polémica sobre el origen de estos vertidos ha puesto en cuestión que el respeto al ciclo integral de nuestras aguas necesita una 
revisión desde la perspectiva de la distribución competencial. Seguramente el caso de este vertido terminará judicializándose, y pasados unos años quizás quede dirimido quien fue la Administración que no cumplió debidamente sus competencias en materia de conservación del estado de las aguas, pero el vertido habrá causado sus nefastas consecuencias ambientales ya sin remedio.

\section{Actuaciones pendientes: reconversión de las minas de mercurio de Almadén y el conflicto en torno a la minería de tierras raras}

El 13 de octubre se ha conocido la importante decisión de la Comisión Europea de Medio Ambiente aprobando el proyecto de reconversión en las minas de mercurio de Almadén, en Ciudad Real, para convertir este mineral en sulfuro de mercurio y posteriormente solidificarlo para su eliminación definitiva. Esta decisión es relevante para nuestra Comunidad Autónoma, pues de acuerdo a la Estrategia Comunitaria del Mercurio adoptada en 2005 por la Comisión Europea, que pretende reducir los niveles de mercurio en el medio ambiente y la exposición del ser humano a dicho elemento, en febrero la Unión Europea había excluido la propuesta inicial desarrollada por Minas de Almadén y Arrayanes (Mayasa) de la Sociedad Estatal de Participaciones Industriales (SEPI) y por el Centro Tecnológico Nacional de Descontaminación del Mercurio, consistente en almacenar el mercurio solidificado. Así se han conseguido validar, frente a las tesis alemanas, las enmiendas más rigurosas presentadas por España respecto a las minas de Almadén, explotadas desde tiempos de los romanos y cuya actividad cesó definitivamente en 2011. Situación que se había complicado tras la prohibición de las exportaciones de mercurio, actividad en la que España era el principal productor internacional. Ahora el Parlamento Europeo deberá negociará el texto definitivo con el Consejo antes de su aprobación definitiva.

Una cuestión que también ha resultado bastante polémica en estos últimos meses ha sido el proyecto minero que la empresa Quantum pretende llevar a cabo en el Campo de Montiel, en Ciudad Real, para la extracción de monacita, uno de los minerales de las "tierras raras". El Informe del Ministerio de Agricultura en la fase previa de consultas de las 3 concesiones solicitadas por la compañía minera considera inviable medioambientalmente este proyecto. Fundamentalmente por encontrarse a 900 metros de la ZEPA “Areas Esteparias del Campo de Montiel”, no abordar aspectos esenciales en proyectos de minería a cielo abierto como los impactos por contaminación difusa o 
vertidos accidentales en las aguas superficiales o subterráneas", y encontrarse en una relevante zona de reproducción para el águila imperial ibérica, en peligro de extinción, o de presencia de lince ibérico.

El texto también alude al informe desfavorable de la Confederación Hidrográfica del Guadiana (CHG) y del Consejo de Seguridad Nuclear (CSN), que asegura que las actividades de explotación "son susceptibles de generar un impacto radiológico sobre los trabajadores, la población y el medio ambiente". 